PROCEEDINGS OF THE

AMERICAN MATHEMATICAL SOCIETY

Volume 130, Number 3, Pages 805-817

S 0002-9939(01)06243-8

Article electronically published on August 28, 2001

\title{
HARMONIC MAPPINGS OF THE SIERPINSKI GASKET TO THE CIRCLE
}

\author{
ROBERT S. STRICHARTZ
}

(Communicated by Christopher D. Sogge)

\begin{abstract}
Harmonic mappings from the Sierpinski gasket to the circle are described explicitly in terms of boundary values and topological data. In particular, all such mappings minimize energy within a given homotopy class. Explicit formulas are also given for the energy of the mapping and its normal derivatives at boundary points.
\end{abstract}

\section{$\S 1$. INTRODUCTION}

Whenever there is a theory of harmonic functions on a space $X$, there should be a theory of harmonic mappings from $X$ to $Y$, where the target space $Y$ is any Riemannian manifold. (See [EL1], EL2], for the standard theory where $X$ is also a Riemannian manifold.) We will be interested in developing such a theory for the class of p.c.f. self-similar fractals with self-similar Dirichlet forms as developed by Kigami [Ki1-Ki4] (see also [S]). A typical example of such a fractal is the Sierpinski gasket SG, and to keep matters simple we will restrict attention to just this case, although it is expected that the methods we develop can be extended more generally. The new elements involving the target space $Y$, in contrast to $\mathbb{R}$, are nontrivial topology and curvature. In this paper we take $Y$ to be the circle, so we have nontrivial topology but no curvature. Numerical computations of mappings with $Y$ equal to the 2-sphere or hyperbolic plane have been done by Gregory Padowski, and may be found at http://mathlab.cit.cornell.edu/ gp36/.

The theory of harmonic functions $h: S G \rightarrow \mathbb{R}$ and the standard self-similar Dirichlet form $\mathcal{E}(u, u)$ is easy to explain in terms of a sequence of graphs $\Gamma_{m}$ approximating SG. We take $\Gamma_{0}$ to be the complete graph on the vertices $V_{0}=\left\{v_{0}, v_{1}, v_{2}\right\}$ of an equilateral triangle in the plane. These vertices will be taken to be the boundary of SG. We define the vertices $V_{m}$ of $\Gamma_{m}$ inductively by $V_{m}=\bigcup_{i=0}^{2} F_{i} V_{m-1}$, where $F_{i} x=\frac{1}{2}\left(x-v_{i}\right)+v_{i}$ make up the iterated function system (i.f.s.) whose attractor is SG. The edge relationship $x \sim_{m} y$ of $\Gamma_{m}$ is given by the condition $x=F_{w} v_{j}$, $y=F_{w} v_{k}$ for some $w, j, k$, where $w=\left(w_{1}, \ldots, w_{m}\right)$ denote a word of length $|w|=m$, and $F_{w}=F_{w_{1}} \circ \cdots \circ F_{w_{m}}$. The energy (or Dirichlet) form $\mathcal{E}(u, u)$ is

Received by the editors September 15, 2000.

2000 Mathematics Subject Classification. Primary 28A80, 58E20.

Key words and phrases. Sierpinski gasket, harmonic mappings, analysis on fractals, self-similar Dirichlet form.

This research was supported in part by the National Science Foundation, Grant DMS 9970337. 
defined by

$$
\mathcal{E}(u, u)=\lim _{m \rightarrow \infty} \mathcal{E}_{m}(u, u)
$$

where

$$
\mathcal{E}_{m}(u, u)=\left(\frac{5}{3}\right)^{m} \sum_{x \sim_{m} y}(u(x)-u(y))^{2} .
$$

The limit always exists in (1.1) because $\mathcal{E}_{m}(u, u)$ is monotone increasing in $m$ for all functions $u$ and is constant for harmonic functions. The domain of $\mathcal{E}$, dom $\mathcal{E}$, is by definition the space of functions for which the limit in (1.1) is finite, and $\mathcal{E}$ may be extended to a bilinear form on dom $\mathcal{E}$ satisfying the axioms of a Dirichlet form. All functions in $\operatorname{dom} \mathcal{E}$ are continuous, a reflection of the fact that points have positive capacity. The space of harmonic functions is 3 -dimensional, each harmonic function $h$ being determined by its boundary values $\left(h\left(v_{0}\right), h\left(v_{1}\right), h\left(v_{2}\right)\right)$ by the local extension algorithm

$$
\left(\begin{array}{l}
h\left(F_{w} v_{0}\right) \\
h\left(F_{w} v_{1}\right) \\
h\left(F_{w} v_{2}\right)
\end{array}\right)=A_{w_{1}} \cdots A_{w_{m}}\left(\begin{array}{l}
h\left(v_{0}\right) \\
h\left(v_{1}\right) \\
h\left(v_{2}\right)
\end{array}\right)
$$

for the matrices

$$
A_{0}=\left(\begin{array}{ccc}
1 & 0 & 0 \\
\frac{2}{5} & \frac{2}{5} & \frac{1}{5} \\
\frac{2}{5} & \frac{1}{5} & \frac{2}{5}
\end{array}\right), \quad A_{1}=\left(\begin{array}{ccc}
\frac{2}{5} & \frac{2}{5} & \frac{1}{5} \\
0 & 1 & 0 \\
\frac{1}{5} & \frac{2}{5} & \frac{2}{5}
\end{array}\right), \quad A_{2}=\left(\begin{array}{ccc}
\frac{2}{5} & \frac{1}{5} & \frac{2}{5} \\
\frac{1}{5} & \frac{2}{5} & \frac{2}{5} \\
0 & 0 & 1
\end{array}\right) .
$$

Harmonic functions minimize energy among all functions with given boundary values, and are characterized by the harmonic condition that $h(x)$ for any nonboundary point $x \in V_{m}$ is the average of its value at the 4 neighboring points in $V_{m}$. For this reason we can make the somewhat stronger assertion that any function that is a critical point for the energy functional under fixed boundary values must be harmonic.

Because $\mathcal{E}_{m}(u, u)$ is independent of $m$ when $u$ is harmonic, we have the simple expression

$$
\mathcal{E}(h, h)=\left(h\left(v_{0}\right)-h\left(v_{1}\right)\right)^{2}+\left(h\left(v_{1}\right)-h\left(v_{2}\right)\right)^{2}+\left(h\left(v_{2}\right)-h\left(v_{0}\right)\right)^{2}
$$

for the energy of a harmonic function. We also have a simple expression for the normal derivatives at the boundary points. In general, the normal derivatives $\partial_{n} u\left(v_{j}\right)$ are defined by

$$
\partial_{n} u\left(v_{j}\right)=\lim _{m \rightarrow \infty}\left(\frac{5}{3}\right)^{m}\left(2 u\left(v_{j}\right)-u\left(F_{j}^{m} v_{j+1}\right)-u\left(F_{j}^{m} v_{j-1}\right)\right)
$$

whenever the limit exists. For harmonic functions the values on the right side of (1.6) are independent of $m$, so the limit exists trivially and

$$
\partial_{n} h\left(v_{j}\right)=2 u\left(v_{j}\right)-u\left(v_{j+1}\right)-u\left(v_{j-1}\right) .
$$

The main goal of this paper is to find the analogs of (1.3), (1.5), and (1.7) for harmonic mappings of SG to a circle.

In general, if $u: S G \rightarrow Y$ is continuous and $Y$ is a Riemannian manifold, we may define the energy $\mathcal{E}(u, u)$ by (1.1) where

$$
\mathcal{E}_{m}(u, u)=\left(\frac{5}{3}\right)^{m} \sum_{x \sim m} d_{Y}(u(x), u(y))^{2}
$$


and $d_{Y}$ denotes the metric on $Y$. By elementary arguments the energy exists and is finite if and only if $u(x)$ in local coordinates consists of functions of finite energy. By definition, a harmonic mapping $h$ is a critical point of the function $\mathcal{E}(u, u)$. More precisely,

$$
\left.\frac{d}{d t} \mathcal{E}\left(u_{t}, u_{t}\right)\right|_{t=0}=0
$$

whenever $u_{t}$ is a path with fixed boundary values and $u_{0}=h$. If $h$ minimizes energy in its homotopy class (with fixed boundary values), then it is harmonic, but there may exist other critical points. We have already observed that this does not happen when $Y=\mathbb{R}$, and we will show that it does not happen for the circle, either.

We will allow circles of arbitrary radius, so $S^{1}=\mathbb{R} / \tau \mathbb{Z}$ for some $\tau>0$. Every continuous function $u: S G \rightarrow S^{1}$ has local lifts $\widetilde{u}: U \rightarrow \mathbb{R}$ for small enough neighborhoods $U$ in $\mathrm{SG}$, and if $u$ is topologically trivial, then we may take $U=S G$. It is easy to see that $u$ is a harmonic mapping if and only if the lifts $\widetilde{u}$ are harmonic functions.

Consider the edges of the triangle $T$ with vertices $\left(v_{0}, v_{1}, v_{2}\right)$. Since each edge is topologically trivial we can find lifts defined on the whole edge and define the increments $(\Delta)_{j}$ along the edge opposite $v_{j}$ by

$$
(\Delta)_{j}=\widetilde{u}\left(v_{j+1}\right)-\widetilde{u}\left(v_{j-1}\right)
$$

for the appropriate lift. Note that we have $(\Delta)_{0}+(\Delta)_{1}+(\Delta)_{2}=k \tau$ for some integer $k$; in fact $k=W(u, T)$, the winding number of the image $u(T)$ in $S^{1}$. More generally, let $k_{w}=W\left(u, T_{w}\right)$ where $T_{w}$ is the triangle $F_{w} T$. (Throughout this paper we omit the subscript $w$ when $w$ is the empty word $\emptyset$.) All but a finite number of the $k_{w}$ are zero, because once $T_{w}$ is small enough the image $u\left(T_{w}\right)$ is not onto $S^{1}$, so it is topologically trivial.

Consider a homotopy class of maps with $u\left(v_{j}\right)$ specified. This determines data $\left\{(\Delta)_{j}, k_{w}\right\}$ subject to the consistency conditions

$$
\begin{gathered}
(\Delta)_{j} \equiv u\left(v_{j+1}\right)-u\left(v_{j-1}\right) \quad \bmod \tau, \\
(\Delta)_{0}+(\Delta)_{1}+(\Delta)_{2}=k \tau,
\end{gathered}
$$

and $k_{w}=0$ except for a finite set of words. Conversely, every such data set determines a homotopy class. It is not hard to see that every homotopy class contains an energy minimizer, using the fact that points have positive capacity so that energy limits are automatically uniform limits and so stay within homotopy classes. We will show directly that each homotopy class contains a unique harmonic mapping by giving an explicit formula for the mapping in terms of the data. In the process we will also find a formula for the normal derivatives. The importance of normal derivatives may be seen as follows.

Consider just harmonic functions. We may localize the definition (1.6) of normal derivatives to a cell $F_{w} S G$ and a vertex $F_{w} v_{j}$ on the boundary of this cell by taking

$$
\lim _{m \rightarrow \infty}\left(\frac{5}{3}\right)^{m+|w|}\left(2 u\left(F_{w} v_{j}\right)-u\left(F_{w} F_{j}^{m} v_{j+1}\right)-u\left(F_{w} F_{j}^{m} v_{j-1}\right)\right) .
$$

Again, it is not necessary to take the limit for harmonic functions. If $F_{w} v_{j}$ is not a boundary point of SG, then it can also be represented as $F_{w^{\prime}} v_{j^{\prime}}$, a boundary point of a different cell $F_{w^{\prime}} S G$. The condition that the function be harmonic at the point is the same as the condition that the sum of the two different normal derivatives be zero. Thus we may glue together harmonic functions defined on neighboring cells 
if the values of the functions are the same and the normal derivatives sum to zero. When we consider harmonic mappings, we may still define normal derivatives by considering lifts, since the differences in (1.13) are independent of the lift chosen. Again the sum of normal derivatives at a nonboundary point must be zero. However, when we glue together different lifts, we must allow jump discontinuities that are integer multiples of $\tau$. Conversely, such a consistent collection of local harmonic functions determines a harmonic mapping. In this way the problem of harmonic mappings $h: S G \rightarrow S^{1}$ is purely topological.

In Section 2 we present the details of the extension algorithm for harmonic mappings, and at the same time compute normal derivatives. In Section 3 we compute the energy in terms of the data, and show that mappings with prescribed boundary values in $S^{1}$ that minimize energy are topologically trivial (all $k_{w}=0$ ). We also give a completely explicit set of formulas in the simple case that $k_{w}=0$ for $|w| \geq 2$.

Harmonic mappings from SG to $\mathbb{R}^{n}$ are characterized by the condition that the coordinate functions be harmonic functions, and the energy is simply the sum of the energies of the coordinate functions. An illustration of such a mapping for $n=2$ may be found in [Ki3]. The results of this paper easily extend to mappings to any flat torus, which may be represented as $\mathbb{R}^{n} / \Lambda^{n}$ for a lattice $\Lambda^{n}$ generated by $n$ linearly independent vectors $\tau_{1}, \ldots, \tau_{n}$. (Unless these vectors are orthogonal, this is not a geometric product of circles.) It is simply a matter of replacing scalar quantities by $n$-vectors, and correctly interpreting certain products. For example, the increments $\left(\Delta_{w}\right)_{j}$ are vectors, and the winding numbers $k_{w}$ are elements of the integer lattice $\mathbb{Z}^{n}$, with the product $\tau k_{w}$ being interpreted as the vector $\left(k_{w}\right)_{1} \tau_{1}+$ $\cdots+\left(k_{w}\right)_{n} \tau_{n}$. Then all the formulas of Section 2 remain valid, with $\left(N_{w}\right)_{j},\left(\lambda_{w j}\right)_{k}$ and $\left(\delta_{w}\right)_{j}$ all being vectors, but $\gamma(v, j)$ remaining the same scalar values. For the results in Section 3, it is necessary to interpret the norm in (3.3) and (3.6) as the Euclidean norm in $\mathbb{R}^{3 n}$, and the square $\left(\tau k_{w}\right)^{2}$ as the $\mathbb{R}^{n}$ dot product $\left(\tau k_{w}\right) \cdot\left(\tau k_{w}\right)$ with $\tau k_{w}$ as above. Note that $\mu_{w}$ is an $n$-vector but $E_{w}$ is still a scalar.

\section{$\S 2$. The EXTENSION ALGORITHM}

In this section we assume that $u\left(v_{j}\right)$ in $S^{1}$ are given, and compatible data $\left\{(\Delta)_{j}, k_{w}\right\}$ are given to determine a homotopy class. Let $h$ denote a harmonic mapping in this class. Rather than give formulas for the values of $h$ at points, we will give formulas for the increments of $h$ along the edges of the triangles. Let

$$
\left(\Delta_{w}\right)_{j}=\widetilde{h}\left(F_{w} v_{j+1}\right)-\widetilde{h}\left(F_{w} v_{j-1}\right)
$$

for any lift $\widetilde{h}$ of $h$ along this edge. (When $w$ is the empty word we write $(\Delta)_{j}$ rather than $\left(\Delta_{\emptyset}\right)_{j}$.) We want an inductive formula that enables us to compute these increments for words of length $m+1$ in terms of increments for words of length $m$, since the data supplies us with the initial values for the empty word. In the topologically trivial case the result is easy.

Lemma 2.1. Suppose all $k_{w}=0$. Then

$$
\left(\begin{array}{c}
\left(\Delta_{w j}\right)_{(j-1)} \\
\left(\Delta_{w j}\right)_{j} \\
\left(\Delta_{w j}\right)_{(j+1)}
\end{array}\right)=\left(\begin{array}{ccc}
\frac{1}{2} & \frac{1}{10} & -\frac{1}{10} \\
-\frac{2}{5} & -\frac{1}{5} & -\frac{2}{5} \\
-\frac{1}{10} & \frac{1}{10} & \frac{1}{2}
\end{array}\right)\left(\begin{array}{c}
\left(\Delta_{w}\right)_{(j-1)} \\
\left(\Delta_{w}\right)_{j} \\
\left(\Delta_{w}\right)_{(j+1)}
\end{array}\right) .
$$


Proof. In this case there exists a global lift $\widetilde{h}$ which is a harmonic function. Thus (1.3) holds for $\widetilde{h}$. An elementary computation shows that (2.2) is equivalent to (1.3).

Note that in this case we always have $\left(\Delta_{w}\right)_{0}+\left(\Delta_{w}\right)_{1}+\left(\Delta_{w}\right)_{2}=0$, so the matrix given in (2.2) is not the only possible choice. One could add an arbitrary constant to each row. This particular matrix was chosen because it has column sums equal to zero.

Our strategy for the general case will be to add a correction term to (2.2). In other words we define $\left(\lambda_{w j}\right)_{k}$ by

$$
\left(\begin{array}{c}
\left(\Delta_{w j}\right)_{(j-1)} \\
\left(\Delta_{w j}\right)_{j} \\
\left(\Delta_{w j}\right)_{(j+1)}
\end{array}\right)=\left(\begin{array}{ccc}
\frac{1}{2} & \frac{1}{10} & -\frac{1}{10} \\
-\frac{2}{5} & -\frac{1}{5} & -\frac{2}{5} \\
-\frac{1}{10} & \frac{1}{10} & \frac{1}{2}
\end{array}\right)\left(\begin{array}{c}
\left(\Delta_{w}\right)_{(j-1)} \\
\left(\Delta_{v}\right)_{j} \\
\left(\Delta_{w}\right)_{(j+1)}
\end{array}\right)+\left(\begin{array}{c}
\left(\lambda_{w j}\right)_{(j-1)} \\
\left(\lambda_{w j}\right)_{j} \\
\left(\lambda_{w j}\right)_{(j+1)}
\end{array}\right)
$$

We will also require similar correction terms for normal derivatives. Write $\left(N_{w}\right)_{j}$ for (1.13). Note that the matching condition on adjacent cells means

$$
\left(N_{w j}\right)_{(j+1)}+\left(N_{w_{(j+1)}}\right)_{j}=0
$$

and we also have the consistency condition

$$
\left(N_{w j}\right)_{j}=\left(N_{w}\right)_{j}
$$

In the topologically trivial case we have simply

$$
\left(N_{w}\right)_{j}=\left(\frac{5}{3}\right)^{|w|}\left(\left(\Delta_{w}\right)_{j-1}-\left(\Delta_{w}\right)_{j+1}\right)
$$

by taking $m=0$ on the right side of (1.13). In the general case we define the correction terms $\left(\delta_{w}\right)_{j}$ by

$$
\left(N_{w}\right)_{j}=\left(\frac{5}{3}\right)^{|w|}\left(\left(\Delta_{w}\right)_{j-1}-\left(\Delta_{w}\right)_{j+1}+\left(\delta_{w}\right)_{j}\right) .
$$

Both correction terms $\left(\lambda_{w}\right)_{j}$ and $\left(\delta_{w}\right)_{j}$ will be zero if $|w|$ is large enough so that $h$ is topologically trivial on $F_{w} S G$.

Our first goal is to translate the condition that $h$ be a harmonic mapping into a system of equations for the correction terms. Then we will solve the equations. The next result might be of independent interest.

Lemma 2.2. For any $w$,

$$
\left(N_{w}\right)_{0}+\left(N_{w}\right)_{1}+\left(N_{w}\right)_{2}=0 .
$$

Proof. This is obvious for harmonic functions, hence it is true for the length of $w$ long enough, say $|w|>m_{0}$. We will show the inductive argument that if (2.8) holds for all words with $|w|=m+1$, it holds when $|w|=m$. Indeed, if $|w|=m$, then by the induction hypothesis $\left(N_{w j}\right)_{0}+\left(N_{w j}\right)_{1}+\left(N_{w j}\right)_{2}=0$ for $j=0,1$, 2. Sum these equations and use (2.4) to eliminate the nondiagonal terms $\left(N_{w j}\right)_{k}$ with $j \neq k$. Because of (2.5) the remaining terms yield (2.8).

Lemma 2.3. The following equations on $\left(\lambda_{w j}\right)_{k}$ and $\left(\delta_{w}\right)_{j}$ are necessary and sufficient for them to be associated with a harmonic mapping in the given hopotopy class:

$$
\left(\lambda_{w(j-1)}\right)_{j}+\left(\lambda_{w(j+1)}\right)_{j}=0
$$




$$
\begin{gathered}
\left(\lambda_{w j}\right)_{0}+\left(\lambda_{w j}\right)_{1}+\left(\lambda_{w j}\right)_{2}=\tau k_{w j}, \\
\left(\lambda_{w(j+1)}\right)_{(j+1)}-\left(\lambda_{w(j+1)}\right)_{(j-1)}+\left(\lambda_{w j}\right)_{(j-1)}-\left(\lambda_{w j}\right)_{j} \\
=\left(\delta_{w j}\right)_{(j+1)}+\left(\delta_{w(j+1)}\right)_{j}, \\
\frac{5}{3}\left(\left(\lambda_{w j}\right)_{(j-1)}-\left(\lambda_{w j}\right)_{(j+1)}+\left(\delta_{w j}\right)_{j}\right)=\left(\delta_{w}\right)_{j}, \\
\left(\delta_{w}\right)_{0}+\left(\delta_{w}\right)_{1}+\left(\delta_{w}\right)_{2}=0,
\end{gathered}
$$

for all $w$ and all $j$.

Proof. Each edge in the triangle $F_{w} T$ splits into a union of two edges of triangles of the next level, yielding the consistency condition

$$
\left(\Delta_{w(j-1)}\right)_{j}+\left(\Delta_{w(j+1)}\right)_{j}=\left(\Delta_{w}\right)_{j} .
$$

Substituting (2.3) and simplifying shows that (2.9) is equivalent to (2.14). The definition of the winding number requires

$$
\left(\Delta_{w j}\right)_{0}+\left(\Delta_{w j}\right)_{1}+\left(\Delta_{w j}\right)_{2}=\tau k_{w j}
$$

which is easily seen to be equivalent to (2.10).

Next we consider conditions involving normal derivatives. Condition (2.8) is clearly equivalent to (2.13). Condition (2.5) after substituting (2.7) becomes

$$
\frac{5}{3}\left(\left(\Delta_{w j}\right)_{(j-1)}-\left(\Delta_{w j}\right)_{(j+1)}+\left(\delta_{w j}\right)_{j}\right)=\left(\Delta_{w}\right)_{(j-1)}-\left(\Delta_{w}\right)_{(j+1)}+\left(\delta_{w}\right)_{j} .
$$

When we substitute (2.3) and simplify, this is equivalent to (2.12). Similarly, condition (2.4) after substituting (2.7) becomes

$$
\begin{aligned}
&\left(\Delta_{w j}\right)_{j}-\left(\Delta_{w j}\right)_{(j-1)}+\left(\Delta_{w(j+1)}\right)_{(j-1)} \\
&-\left(\Delta_{w(j+1)}\right)_{(j+1)}+\left(\delta_{w j}\right)_{(j+1)}+\left(\delta_{w(j+1)}\right)_{j}=0,
\end{aligned}
$$

and after substituting (2.3) and simplifying, this is equivalent to (2.11).

Thus the conditions are necessary (in fact (2.13) could be eliminated since it follows from (2.11) and (2.12) as in the proof of Lemma 2.2). But conversely, if the conditions are satisfied, solving (2.3) yields a mapping in the required homotopy class that has lifts on cells $F_{w} S G$ for all sufficiently large words that are harmonic functions, and satisfies the required matching condition on normal derivatives on adjacent cells $F_{w} S G$. Thus it is a harmonic mapping.

Lemma 2.4. The unique solution of (2.9), (2.10) and (2.11) for $\left(\lambda_{w j}\right)_{k}$ in terms of $\left(\delta_{w j}\right)_{k}$ is given by

$$
\begin{gathered}
\left(\lambda_{w j}\right)_{(j+1)}=\frac{1}{5} \tau\left(k_{w j}-k_{w(j-1)}\right)-\frac{3}{10}\left(\left(\delta_{w j}\right)_{(j-1)}+\left(\delta_{w(j-1)}\right)_{j}\right) \\
-\frac{1}{10}\left(\left(\delta_{w j}\right)_{(j+1)}+\left(\delta_{w(j+1)}\right)_{j}+\left(\delta_{w(j+1)}\right)_{(j-1)}+\left(\delta_{w(j+1)}\right)_{j}\right), \\
\left(\lambda_{w j}\right)_{(j-1)}=\frac{1}{5} \tau\left(k_{w j}-k_{w(j+1)}\right)+\frac{3}{10}\left(\left(\delta_{w j}\right)_{(j+1)}+\left(\delta_{w(j+1)}\right)_{j}\right) \\
+\frac{1}{10}\left(\left(\delta_{w j}\right)_{(j-1)}+\left(\delta_{w(j-1)}\right)_{j}+\left(\delta_{w(j-1)}\right)_{(j+1)}+\left(\delta_{w(j+1)}\right)_{(j-1)}\right),
\end{gathered}
$$




$$
\begin{aligned}
\left(\lambda_{w j}\right)_{j}=\frac{1}{5} & \tau\left(3 k_{w j}+k_{w(j-1)}+k_{w(j+1)}\right)+\frac{1}{5}\left(\left(\delta_{w j}\right)_{(j-1)}+\left(\delta_{w(j-1)}\right)_{j}\right) \\
& -\frac{1}{5}\left(\left(\delta_{w j}\right)_{(j+1)}+\left(\delta_{w(j+1)}\right)_{j}\right) .
\end{aligned}
$$

Proof. Note that for fixed $w$ we have 9 linear equations in 9 unknowns. First we use (2.10) to solve for the diagonal variables $\left(\lambda_{w j}\right)_{j}$ in terms of the others:

$$
\left(\lambda_{w j}\right)_{j}=\tau k_{w j}-\left(\lambda_{w j}\right)_{(j+1)}-\left(\lambda_{w j}\right)_{(j-1)} .
$$

We substitute (2.19) into (2.11) to obtain

$$
\begin{aligned}
\left(\lambda_{w j}\right)_{(j+1)} & +\left(\lambda_{w(j+1)}\right)_{j}+2\left(\left(\lambda_{w j}\right)_{(j-1)}+\left(\lambda_{w(j+1)}\right)_{(j-1)}\right) \\
& =\tau\left(k_{w j}-k_{w(j+1)}\right)+\left(\delta_{w j}\right)_{(j+1)}+\left(\delta_{w(j+1)}\right)_{j} .
\end{aligned}
$$

Now (2.9) and (2.20) give 6 equations in 6 unknowns, and it is easy to check that (2.16) and (2.17) give the unique solutions. Substituting these into (2.19) yields (2.18).

Lemma 2.5. The equations (2.9)-(2.13) have a unique solution when the $\lambda$ 's are given by (2.16)-(2.18) and the $\delta$ 's are determined by

$$
\begin{aligned}
\left(\delta_{w}\right)_{j}=\frac{1}{3} & \tau\left(k_{w(j-1)}-k_{w(j+1)}\right)+\left(\delta_{w j}\right)_{j}+\frac{2}{3}\left(\left(\delta_{w(j+1)}\right)_{j}+\left(\delta_{w(j-1)}\right)_{j}\right) \\
+ & \frac{1}{3}\left(\left(\delta_{w(j+1)}\right)_{(j-1)}+\left(\delta_{w(j-1)}\right)_{(j+1)}\right) .
\end{aligned}
$$

Proof. Since $\left(\delta_{w}\right)_{j}=0$ for $w$ long enough, it is clear that (2.21) allows us to solve inductively for all words working down in length. We obtain (2.21) by substituting (2.16)-(2.18) into (2.12) to eliminate the $\lambda$ 's, and then simplifying using (2.13). We can then prove (2.13) inductively directly from (2.21), hence (2.21) implies (2.12).

Lemma 2.6. The solution to (2.21) is given by the finite sum

$$
\left(\delta_{w}\right)_{j}=\sum_{v \neq \emptyset} \tau 3^{-|v|} \gamma(v, j) k_{w v},
$$

where $\gamma(v, j)$ are combinatorial coefficients given explicitly by

$$
\left(\begin{array}{l}
\gamma(v, 0) \\
\gamma(v, 1) \\
\gamma(v, 2)
\end{array}\right)=M_{v_{1}} M_{v_{2}} \cdots M_{v_{m-1}} e_{v_{m}}
$$

where

$$
M_{0}=\left(\begin{array}{lll}
3 & 0 & 0 \\
0 & 2 & 1 \\
0 & 1 & 2
\end{array}\right), \quad M_{1}=\left(\begin{array}{lll}
2 & 0 & 1 \\
0 & 3 & 0 \\
1 & 0 & 2
\end{array}\right), \quad M_{2}=\left(\begin{array}{lll}
2 & 1 & 0 \\
1 & 2 & 0 \\
0 & 0 & 3
\end{array}\right)
$$

and

$$
e_{0}=\left(\begin{array}{c}
0 \\
1 \\
-1
\end{array}\right), \quad e_{1}=\left(\begin{array}{c}
-1 \\
0 \\
1
\end{array}\right), \quad e_{2}=\left(\begin{array}{c}
1 \\
-1 \\
0
\end{array}\right)
$$


Proof. From the form of (2.21) it is clear that the solution must have the form (2.22) for some coefficients $\gamma(v, j)$. Substituting (2.22) into (2.21) yields

$$
\begin{aligned}
\sum_{v \neq \emptyset} \tau 3^{-|v|} \gamma(v, j) k_{w v} \\
=\frac{1}{3} \tau k_{w(j-1)}-\frac{1}{3} \tau k_{w(j+1)}+\sum_{v \neq \emptyset} 3^{-|v|} \tau \gamma(v, j) k_{w j v} \\
\quad+\frac{2}{3} \sum_{v \neq \emptyset} 3^{-|v|} \tau \gamma(v, j)\left(k_{w(j+1) v}+k_{w(j-1) v}\right) \\
\quad+\frac{1}{3} \sum_{v \neq \emptyset} 3^{-|v|} \tau\left(\gamma(v, j+1) k_{w(j-1) v}+\gamma(v, j-1) k_{w(j+1) v}\right) .
\end{aligned}
$$

Equating coefficients of the same $k$ 's yields the initial values

$$
\gamma(j, j)=0, \quad \gamma(j-1, j)=1, \quad \gamma(j+1, j)=-1
$$

and the recursion relations

$$
\left(\begin{array}{c}
\gamma((j-1) v, j) \\
\gamma(j v, j) \\
\gamma((j+1) v, j)
\end{array}\right)=\left(\begin{array}{lll}
0 & 2 & 1 \\
0 & 3 & 0 \\
1 & 2 & 0
\end{array}\right)\left(\begin{array}{c}
\gamma(v, j-1) \\
\gamma(v, j) \\
\gamma(v, j+1)
\end{array}\right) .
$$

We can rewrite $(2.28)$ as

$$
\left(\begin{array}{l}
\gamma(j v, 0) \\
\gamma(j v, 1) \\
\gamma(j v, 2)
\end{array}\right)=M_{j}\left(\begin{array}{l}
\gamma(v, 0) \\
\gamma(v, 1) \\
\gamma(v, 2)
\end{array}\right)
$$

with $M_{j}$ given by (2.24), which yields (2.23).

Note that (2.22) is a local formula, in the sense that the normal derivatives in the cell $F_{w} S G$ are determined by the topological data of winding numbers for cycles contained in that cell alone. The same is true for the $\left(\lambda_{w j}\right)_{k}$ in view of Lemma 2.4. We summarize our results as follows.

Theorem 2.7. There is a unique harmonic mapping in each homotopy class, and its values are determined by the increments $\left(\Delta_{w}\right)_{j}$ along edges in $F_{w} S G$. These increments for $w=\emptyset$ are given by the initial data, and then (2.3) determines them inductively (with the length of $w$ increasing), where $\left(\lambda_{w j}\right)_{k}$ are given by (2.16)(2.18) and $\left(\delta_{w}\right)_{j}$ are given by (2.22). In particular, the normal derivatives at the boundary points are given by

$$
\partial_{n} h\left(v_{j}\right)=(\Delta)_{j-1}-(\Delta)_{j+1}+\sum_{v \neq \emptyset} \tau 3^{-|v|} \gamma(v, j) k_{v} .
$$

Corollary 2.8. There exist nonconstant harmonic mappings satisfying Neumann conditions $\partial_{n} h\left(v_{j}\right)=0$ at all the boundary points.

Proof. We just have to arrange for (2.30) to equal zero. A simple example has all $k_{v}=0$ for $v \neq \emptyset, k=1$ and $(\Delta)_{0}=(\Delta)_{1}=(\Delta)_{2}=\tau / 3$. 


\section{§3. ENERGy COMPUtATIONS}

In this section we compute the energy $\mathcal{E}(h, h)=\mathcal{E}$ of a harmonic mapping in terms of the data. In fact

$$
\mathcal{E}=\sum_{|w|=m} \mathcal{E}_{w}
$$

for each $m$, where $\mathcal{E}_{w}$ denotes the contribution toward the energy from the cell $F_{w} S G$. When $m$ is large enough

$$
\mathcal{E}_{w}=\left(\frac{5}{3}\right)^{m}\left(\left(\Delta_{w}\right)_{0}^{2}+(\Delta w)_{1}^{2}+\left(\Delta_{w}\right)_{2}^{2}\right)=\left(\frac{5}{3}\right)^{m}\left\|\Delta_{w}\right\|^{2},
$$

where we use vector notation $\Delta_{w}=\left(\left(\Delta_{w}\right)_{0},\left(\Delta_{w}\right)_{1},\left(\Delta_{w}\right)_{2}\right)$ and \|\| stands for the Euclidean norm. We seek an expression of the form

$$
\mathcal{E}_{w}=\left(\frac{5}{3}\right)^{m}\left(\left\|\Delta_{w}+\mu_{w}\right\|^{2}+E_{w}\right)
$$

for any $w$, where $\mu_{w}$ and $E_{w}$ are vector and scalar correction terms involving only the topological data. Note that (3.3) does not determine $\mu_{w}$ and $E_{w}$ uniquely, since by $(2.15)$ we may add an arbitrary multiple of the constant vector $(1,1,1)$ to $\mu_{w}$ and compensate by adjusting $E_{w}$.

Theorem 3.1. The energy $\mathcal{E}_{w}$ is given by (3.3) for

$$
\mu_{w}=\sum_{v \neq \emptyset} B_{v_{1}} \cdots B_{v_{m}} \lambda_{w v}
$$

where

$$
B_{0}=\left(\begin{array}{ccc}
-\frac{1}{3} & \frac{1}{6} & \frac{1}{6} \\
-\frac{2}{3} & \frac{5}{6} & -\frac{1}{6} \\
-\frac{2}{3} & -\frac{1}{6} & \frac{5}{6}
\end{array}\right), B_{1}=\left(\begin{array}{ccc}
\frac{5}{6} & -\frac{2}{3} & -\frac{1}{6} \\
\frac{1}{6} & -\frac{1}{3} & \frac{1}{6} \\
-\frac{1}{6} & -\frac{2}{3} & \frac{5}{6}
\end{array}\right), B_{2}=\left(\begin{array}{ccc}
\frac{5}{6} & -\frac{1}{6} & -\frac{2}{3} \\
-\frac{1}{6} & \frac{5}{6} & -\frac{2}{3} \\
\frac{1}{6} & \frac{1}{6} & -\frac{1}{3}
\end{array}\right)
$$

and

$$
\begin{aligned}
E_{w}=-\left\|\mu_{w}\right\|^{2} & +\frac{1}{2}\left(\tau k_{w}\right)^{2}+\frac{1}{2} \sum_{v \neq \emptyset}\left(\frac{5}{3}\right)^{|v|}\left(\tau k_{w v}\right)^{2} \\
& +\sum_{v \neq \emptyset}\left(\frac{5}{3}\right)^{|v|}\left(\left\|\mu_{w v}+\lambda_{w v}\right\|^{2}-\left\|\mu_{w v}\right\|^{2}\right) .
\end{aligned}
$$

Proof. Since

$$
\mathcal{E}_{w}=\mathcal{E}_{w_{0}}+\mathcal{E}_{w_{1}}+\mathcal{E}_{w_{2}}
$$

we substitute (3.3) in (3.7) and attempt to obtain recursion relations for the correction terms. We obtain first

$$
\left\|\Delta_{w}+\mu_{w}\right\|^{2}+E_{w}=\frac{5}{3} \sum_{j=0}^{2}\left\|\Delta_{w j}+\mu_{w j}\right\|^{2}+\sum_{j=0}^{2} E_{w j},
$$

and then we substitute (2.3) into the right side of (3.8) to eliminate $\Delta_{w j}$. We claim that the quadratic terms in $\Delta_{w}$ are the same on both sides of (3.8). Indeed, on the 
left side we have $\left\|\Delta_{w}\right\|^{2}$, while on the right side we have

$$
\begin{aligned}
& \frac{5}{3} \sum_{j=0}^{2}\left\|\Delta_{w j}\right\|^{2}=\frac{5}{3} \sum_{j=0}^{2}\left(\left(-\frac{1}{5}\left(\Delta_{w}\right)_{j}-\frac{2}{5}\left(\Delta_{w}\right)_{(j+1)}-\frac{2}{5}\left(\Delta_{w}\right)_{(j-1)}\right)^{2}\right. \\
&+\left(\frac{1}{10}\left(\Delta_{w}\right)_{j}+\frac{1}{2}\left(\Delta_{w}\right)_{(j+1)}-\frac{1}{10}\left(\Delta_{w}\right)_{(j-1)}\right)^{2} \\
&\left.+\left(\frac{1}{10}\left(\Delta_{w}\right)_{j}-\frac{1}{10}\left(\Delta_{w}\right)_{(j+1)}+\frac{1}{2}\left(\Delta_{w}\right)_{(j-1)}\right)^{2}\right) \\
&+ \text { lower order terms } \\
&=\left\|\Delta_{w}\right\|^{2}+\frac{1}{2}\left(\left(\Delta_{w}\right)_{0}+\left(\Delta_{w}\right)_{1}+\left(\Delta_{w}\right)_{2}\right)^{2}+\text { lower order terms } \\
&=\left\|\Delta_{w}\right\|^{2}+\frac{1}{2}\left(\tau k_{w}\right)^{2}+\text { lower order terms. }
\end{aligned}
$$

Next we equate the terms in (3.8) that are linear in $\Delta_{w}$ to obtain

$$
\begin{aligned}
& \sum_{j=0}^{2}\left(\Delta_{w}\right)_{j}\left(\mu_{w}\right)_{j} \\
& =\frac{5}{3} \sum_{j=0}^{2}\left(\left(\left(\mu_{w j}\right)_{j}+\left(\lambda_{w j}\right)_{j}\right)\left(-\frac{1}{5}\left(\Delta_{w}\right)_{j}-\frac{2}{5}\left(\Delta_{w}\right)_{(j+1)}-\frac{2}{5}\left(\Delta_{w}\right)_{(j-1)}\right)\right. \\
& \quad+\left(\left(\mu_{w j}\right)_{(j+1)}+\left(\lambda_{w j}\right)_{(j+1)}\right)\left(\frac{1}{10}\left(\Delta_{w}\right)_{j}+\frac{1}{2}\left(\Delta_{w}\right)_{(j+1)}-\frac{1}{10}\left(\Delta_{w}\right)_{(j-1)}\right) \\
& \left.\quad+\left(\left(\mu_{w j}\right)_{(j-1)}+\left(\lambda_{w j}\right)_{(j-1)}\right)\left(\frac{1}{10}\left(\Delta_{w}\right)_{j}-\frac{1}{10}\left(\Delta_{w}\right)_{(j+1)}+\frac{1}{2}\left(\Delta_{w}\right)_{(j-1)}\right)\right) .
\end{aligned}
$$

Equating separately the factors of $\left(\Delta_{w}\right)_{j}$ yields the vector equation

$$
\mu_{w}=\sum_{j=0}^{2} B_{j}\left(\mu_{w j}+\lambda_{w j}\right)
$$

where $B_{j}$ are given by (3.5). Equating everything that remains in (3.8) yields

$$
E_{w}=-\left\|\mu_{w}\right\|^{2}+\frac{1}{2}\left(\tau k_{w}\right)^{2}+\frac{5}{3} \sum_{j=0}^{2} E_{w j}+\frac{5}{3} \sum_{j=0}^{2}\left\|\mu_{w j}+\lambda_{w j}\right\|^{2} .
$$

Altogether we have shown that a solution of (3.9) and (3.10) gives a solution of (3.8), hence a valid formula of the form (3.3). But it is straightforward to show that (3.4) solves (3.9) and then (3.6) solves (3.10).

Example 3.2. Because the formulas are so complicated in the general case, it is worth examining in detail the special case in which the topological data $k_{w}$ are all zero for $|w| \geq 2$. So we are given $(\Delta)_{0},(\Delta)_{1},(\Delta)_{2}, k, k_{0}, k_{1}, k_{2}$ subject to the condition

$$
(\Delta)_{0}+(\Delta)_{1}+(\Delta)_{2}=k \tau .
$$

The mapping is uniquely determined once we specify the value at one of the boundary points, say $u\left(v_{0}\right)$, but all our formulas are in terms of increments so this value does not enter into consideration. 
We have $\delta_{w}=0$ and $\lambda_{w}=0$ if $|w| \geq 2$ because at that level the mapping is topologically trivial. By (2.21) we see that $\delta_{w}=0$ also for $|w|=1$, and

$$
\delta=\frac{\tau}{3}\left(\begin{array}{l}
k_{2}-k_{1} \\
k_{0}-k_{2} \\
k_{1}-k_{0}
\end{array}\right),
$$

where $w=\emptyset$ (we write $\delta$ for $\delta_{\emptyset}$ ). By (2.16)-(2.18) we have

$$
\begin{aligned}
\lambda_{0} & =\frac{\tau}{5}\left(\begin{array}{c}
3 k_{0}+k_{1}+k_{2} \\
k_{0}-k_{2} \\
k_{0}-k_{1}
\end{array}\right), \quad \lambda_{1}=\frac{\tau}{5}\left(\begin{array}{c}
k_{1}-k_{2} \\
k_{0}+3 k_{1}+k_{2} \\
k_{1}-k_{0}
\end{array}\right), \\
\lambda_{2} & =\frac{\tau}{5}\left(\begin{array}{c}
k_{2}-k_{1} \\
k_{2}-k_{0} \\
k_{0}+k_{1}+3 k_{2}
\end{array}\right) .
\end{aligned}
$$

By (3.4) we have $\mu_{w}=0$ for $|w| \geq 1$ and $\mu=B_{0} \lambda_{0}+B_{1} \lambda_{1}+B_{2} \lambda_{2}$ or

$$
\mu=-\frac{\tau}{3}\left(\begin{array}{c}
k_{0}+2 k_{1}+2 k_{2} \\
2 k_{0}+k_{1}+2 k_{2} \\
2 k_{0}+2 k_{1}+k_{2}
\end{array}\right) .
$$

By (3.6) we have

$$
E_{j}=\frac{1}{2} \tau^{2} k_{j}^{2}
$$

and

$$
\begin{aligned}
E & =-\|\mu\|^{2}+\frac{1}{2} \tau^{2} k^{2}+\frac{5}{6} \tau^{2}\left(k_{0}^{2}+k_{1}^{2}+k_{2}^{2}\right) \\
& =\frac{\tau^{2}}{18}\left(9 k^{2}+25\left(k_{0}^{2}+k_{1}^{2}+k_{2}^{2}\right)-10\left(k_{0}+k_{1}+k_{2}\right)^{2}\right) .
\end{aligned}
$$

Therefore, the extension algorithm computes $\left(\Delta_{j}\right)_{k}$ via (2.3) explicitly as

$$
\begin{aligned}
\left(\begin{array}{c}
\left(\Delta_{j}\right)_{(j-1)} \\
\left(\Delta_{j}\right)_{j} \\
\left(\Delta_{j}\right)_{(j+1)}
\end{array}\right)= & \left(\begin{array}{ccc}
\frac{1}{2} & \frac{1}{10} & -\frac{1}{10} \\
-\frac{2}{5} & -\frac{1}{5} & -\frac{2}{5} \\
-\frac{1}{10} & \frac{1}{10} & \frac{1}{2}
\end{array}\right)\left(\begin{array}{c}
(\Delta)_{(j-1)} \\
(\Delta)_{j} \\
(\Delta)_{(j+1)}
\end{array}\right) \\
& +\frac{\tau}{5}\left(\begin{array}{c}
3 k_{j}+k_{j-1}-k_{j+1} \\
k_{j}-k_{j-1}
\end{array}\right)
\end{aligned}
$$

and then $\Delta_{w}$ for $|w| \geq 2$ inductively by (2.2). The normal derivatives at the boundary points are given by

$$
\partial_{n} h\left(v_{j}\right)=(N)_{j}=(\Delta)_{(j-1)}-(\Delta)_{(j+1)}+\frac{\tau}{3}\left(k_{j-1}-k_{j+1}\right) .
$$


The total energy is given by

$$
\begin{aligned}
\mathcal{E}(h, h)= & \frac{\tau^{2}}{18}\left(9 k^{2}+25\left(k_{0}^{2}+k_{1}^{2}+k_{2}^{2}\right)-10\left(k_{0}+k_{1}+k_{2}\right)^{2}\right) \\
& +\sum_{j=0}^{2}\left((\Delta)_{j}-\frac{\tau}{3}\left(k_{j}+2 k_{j-1}+2 k_{j+1}\right)\right)^{2} \\
= & \sum_{j=0}^{2}(\Delta)_{j}^{2}+\frac{2}{3} \tau \sum_{j=0}^{2} k_{j}(\Delta)_{j} \\
& +\tau^{2}\left(\frac{1}{2} k^{2}+\frac{3}{2}\left(k_{0}^{2}+k_{1}^{2}+k_{2}^{2}\right)+\frac{1}{3}\left(k_{0}+k_{1}+k_{2}\right)^{2}\right. \\
& \left.\quad-\frac{4}{3} k\left(k_{0}+k_{1}+k_{2}\right)\right) .
\end{aligned}
$$

Theorem 3.3. Given $h\left(v_{j}\right)=x_{j}$ for points $x_{j} \in S^{1}$, the harmonic mapping that minimizes energy is topologically trivial.

Proof. Suppose first that all $k_{w}=0$ for $|w|=1$. Then (3.19) simplifies to

$$
\mathcal{E}(h, h)=\sum_{j=0}^{2}(\Delta)_{j}^{2}+\frac{1}{2}(\tau k)^{2} .
$$

If $k=0$ this is minimized by choosing the two smallest sides $d\left(x_{j}, x_{j+1}\right)$, say $d\left(x_{0}, x_{1}\right)=a$ and $d\left(x_{1}, x_{2}\right)=b$, and taking $(\Delta)_{2}=a,(\Delta)_{0}=b,(\Delta)_{1}=-(a+b)$. This gives the energy $a^{2}+b^{2}+(a+b)^{2}$, and we claim this is the minimum. The only realistic competition is $k=1,(\Delta)_{2}=a,(\Delta)_{0}=b,(\Delta)_{1}=\tau-(a+b)$ with energy

$$
a^{2}+b^{2}+(\tau-(a+b))^{2}+\frac{1}{2} \tau^{2}=a^{2}+b^{2}+(a+b)^{2}+\frac{3}{2} \tau^{2}-2 \tau(a+b) .
$$

Since $\frac{3}{2} \tau^{2}-2 \tau(a+b)>0$ for $(a+b)<3 \tau / 4$ (and this holds because we chose the two smallest sides) this eliminates the competitors. Other competitors have even higher energy.

In general, let $m$ be the largest value of $|w|$ for which $k_{w} \neq 0$. Applying the above argument to the restriction of $h$ to $F_{w} S G$ shows that we could reduce the energy by modifying $h$ on $F_{w} S G$ to make $k_{w}=0$.

\section{REFERENCES}

[EL1] J. Eells and L. Lemaire, A report on harmonic maps, Bull. London Math. Soc. 10 (1978), 1-68. MR 82b:58033

[EL2] J. Eells and L. Lemaire, Another report on harmonic maps, Bull. London Math. Soc. 20 (1988), 385-524. MR 89i:58027

[Ki1] J. Kigami, A harmonic calculus on the Sierpinski spaces, Japan J. Appl. Math. 8 (1989), 259-290. MR 91g:31005

[Ki2] J. Kigami, Harmonic calculus on p.c.f. self-similar sets, Trans. Amer. Math. Soc. 335 (1993), 721-755. MR 93d:39008

[Ki3] J. Kigami, Harmonic metric and Dirichlet form on the Sierpinski gasket, Asymptotic problems in probability theory: stochastic models and diffusions on fractals (K. D. Elworthy and N. Ikeda, eds.), Pitman Research Notes in Math., vol 283, Longman, 1993, pp. 201-218. MR 96m:31014 
[Ki4] J. Kigami, Analysis on Fractals, Cambridge University Press, 2001.

[S] R. S. Strichartz, Analysis on fractals, Notices AMS 46 (1999), 1199-1208.

Department of Mathematics, Malott Hall, Cornell University, Ithaca, New York 14853

E-mail address: str@math.cornell.edu 